\title{
Tackling scale in biomedical flows
}

\author{
C. S. König · M. W. Collins
}

Published online: 20 March 2014

(C) Springer-Verlag Berlin Heidelberg 2014

Fluid flow problems in the areas of biomedical engineering and biomechanics are being addressed at increasingly small scales. This is very apparent in the domain of theoretical modeling where multiscale modeling approaches are steadily on the rise. In addition to utilizing standard computational fluid mechanics models at reduced scales, increasingly lattice Boltzmann and molecular dynamics models are employed to lower the level of these challenging scales even further. For example, multicoupled, multiscale modeling strategies are essential to successfully address the mechanobiologic problem of atherosclerotic plaque initiation at the vascular endothelium where particulates like blood cells as well as biomolecules are involved in the mechanotransductive processes across the endothelial surface layer into the underlying tissue. Similarly, in the field of experimental biofluid mechanics, the wealth of more recently developed imaging techniques and not least of ultrafine optical measurement techniques allow for investigation at the involved small length scales and have set the research bar notably higher of late.

The idea for a special issue addressing scale issues in biomedical flows was seeded as a result of the 2011 Conference on Micro and Nano Flows (MNF2011), held in Thessaloniki, Greece, August 22-24, which was the third, suc-

C. S. König $(\varangle)$

Brunel Institute for Bioengineering, Brunel University,

London, UK

e-mail: Carola.Koenig@brunel.ac.uk

M. W. Collins

School of Engineering and Design, Brunel University, London, UK cessful run of the series. The MNF conference series is unique in the sense that it provides a forum for scientists and engineers working on small-scale flows in the areas of both biofluid mechanics and thermofluids engineering to present their recent research findings. It therefore allows for unique interdisciplinary exchange in aid to address issues that need resolving in progressing the understanding of microand nanoflows while providing a platform for synergy and crossover opportunities between the areas of biomedicine and engineering. This uniqueness was nicely illustrated in the plenary lecture given by Professor George E. Karnidakis from Brown University, USA, entitled "Multiscale modeling of physical and biological systems."

In addition to the regular contributions for the biomedical stream, the conference accommodated an Expert Biomedical Overview Session, the papers of which formed the core of a book entitled "Micro and Nano Flow Systems for Bioanalysis" in the Bioanalysis series edited by Tuan Vo-Dinh also by Springer.

This BMMB special issue was to give presenters of the regular biomedical stream opportunity to publish their extended papers. To round the issue and widen the breadth of the papers, the issue was supplemented by invited papers by international experts in the field. We are extremely grateful to the Editors-in-Chief and the Editorial Board to support our proposal for this special issue on behalf of Springer. Our sincere thank goes to the Editor-in-Chief Professor Gerhard Holzapfel for his guidance on handling on some of the more challenging editorial issues.

This now gives us opportunity to thank also all the authors that submitted their manuscripts for review for this special issue and the reviewers for their efforts to ensure that BMMB's high publication standard was maintained. Particular credit goes to the contributing authors and their topics that passed the review. The latter ranges from blood rheology 
to mass transport across the endothelial glycocalyx layer as well as its shear stress induced redistribution over to blood and interstitial flow in the liver as well as biomedical appli- cations like stents and oxygenators. We trust this issue to be a valuable source of recent research in the area of small-scale biomedical flows for the readership of BMMB. 